\title{
Extraction of Vibration Parameters from Optical Feedback Interferometry Signals using Wavelets
}

\author{
Ajit Jha*, ${ }^{1}$ Francisco J. Azcona, ${ }^{1}$ Carlos Yañez, ${ }^{1}$ and Santiago Royo ${ }^{1}$ \\ ${ }^{1}$ Centre for Sensors, Instruments and and Systems Development (CD6), \\ UPC-BarcelonaTech, Rambla Sant Nebridi 10, Terrassa 08222, Barcelona, Spain*
}

compiled: July 24, 2015

\begin{abstract}
This paper proposes the use of the wavelet transform as a technique well-suited for fringe detection and analysis of optical feedback interferometry (OFI) signals, allowing the retrieval of extremely small physical motion phenomena. A novel algorithm based on wavelet transform is used to process the OFI signal simultaneously in the time and frequency domain, enabling a precise detection of signal fringes and thus, the extraction of amplitude features of the vibrating target with an average error in the order of $0.1 \lambda$. Furthermore, using a complex Morlet wavelet as analyzing wavelet enables to extract important information from the time envelope of the OFI signal. Such envelope can be useful in detecting fringes even in the presence of speckle reducing the error in a displacement reconstruction. Examples of OFI applications including nanometric displacement sensing without direction ambiguity, as well as the measurement of the frequency and velocity of vibrating targets, and the detection in time of non-periodic events are also presented using this wavelet approach. The paper, thus presents a novel approach to the processing of optical feedback interferometry signals.
\end{abstract}

OCIS codes: (120.2650) Fringe analysis; (120.3180) Interferometry; (140.3520) Lasers, injection-locked; (030.6140) Speckle; (110.7410) Wavelets.

http://dx.doi.org/10.1364/XX.99.099999

\section{Introduction}

The extraction of a desired physical quantity with the highest possible precision in spatial and/or time modulated signals has been a common issue in all kind of sensing techniques, and so it is in the case of optical feedback interferometry $(O F I)$. The response of a laser diode $(L D)$ to optical feedback has been investigated since the invention of the laser itself. The dynamic behaviour of LDs with optical feedback has been a subject of interest because it can induce bi-stability, oscillations and chaos. An OFI interferometer is basically a system in which a LD acts as a source, interfering media and detector all in one. The OFI effect is caused when a fraction of the light emitted by a laser (normally in the order of $10^{-7}$ to $10^{-2}$ ) upon a vibrating target returns back into the laser cavity. The backscattered field interferes with the existent standing wave and changes the carrier density, giving rise to a change in refractive index and gain of the material in the

\footnotetext{
* Corresponding author: ajit.jha@cd6.upc.edu
}

active region of the $\mathrm{LD}$. This results in a variation of the frequency of the free-running laser as the gain shifts towards another dominant mode and emission phase. The relation between field and phase is called phase amplitude coupling and is linked to the linewidth enhancement factor $\alpha$ well described in $[1,2]$. A more detailed theoretical and mathematical model of OFI can be found in [1]-[3].

In order to have a better understanding of the OFI phenomenon, let us consider the classic OFI setup presented in Fig.1 where a target is placed at a distance $L_{o}$ from a $L D$. When the target vibrates with a trajectory $A_{t}(t)=A \cos \left(2 \pi f_{t} t\right)$, the optical path between laser and the target varies and can be expressed as $L_{\text {ext }}(t)=L_{o}+A_{t}(t)$. This change also affects the round trip time of the laser beam, which is defined as $\tau_{\text {ext }}(t)=2 L_{\text {ext }}(t) / c$ with $c$ as the speed of light. Because of the target motion, the injected field induces changes in the emitted frequency $f_{f}(t)$ and phase $\phi_{f}(t)$ relative to the free running emission frequency $f_{o}$ of the laser because of the Doppler shift. Mathematically, the phase and power of the field subject to optical feedback can be 


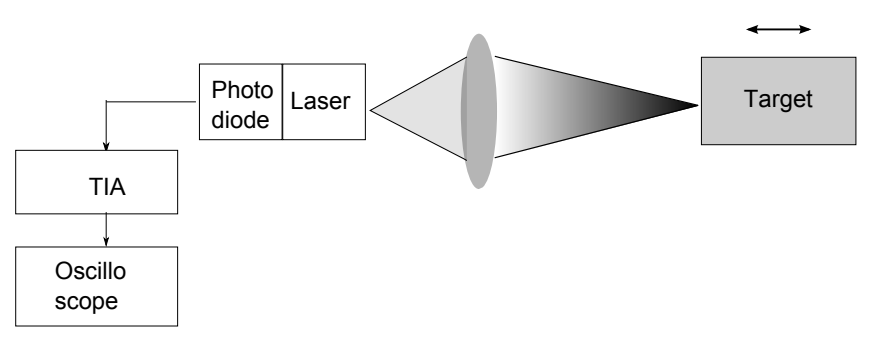

Figure 1. OFI based laser vibrometry.

written as [3]

$$
\begin{aligned}
\phi_{f}(t) & =\phi_{o}(t)-C \sin \left(\phi_{f}(t)+\arctan \alpha\right) \\
P_{f}(t) & =P_{o}\left[1+m F\left(\phi_{f}(t)\right)\right] \\
F\left(\phi_{f}(t)\right) & =\cos \left(2 \pi f_{f}(t) \tau_{\text {ext }}(t)\right)
\end{aligned}
$$

where $\phi_{f}(t)$ and $\phi_{o}(t)$ are the lasing phases for the feedback and free-running lasers, respectively, $P_{f}(t)$ and $P_{o}(t)$ are the optical output power under feedback and standalone laser conditions, $m$ is a modulation index (typically in the order of $10^{-3}$ ) [4] and $C$ is the feedback parameter $[5,6]$ which determines the shape of the OFI signal and the number of modes involved. From Eq.(1) and Eq.(2), it is clear that the OFI signal depends upon $C$. For the proposed methodology, it is desirable that $C$ is always less or close to a value of 1 . For values of $C \approx 1$, the OFI signal has sawtooth-like waveform where the direction of the target can be found by evaluating the signal slope before each transition, and where each transition represents an increment of the amplitude in the defined direction by halfwavelength $(\lambda / 2)$. Experimental details on the extraction of $C$ from OFI signals can be found in [7].

The determination of the displacement of the target by counting the fringes appearing in the signal was proposed by Donati et. al [8]. The accuracy of the method depends upon the precise determination of the position and number of fringes, which are often corrupted by external factors such us white noise, impulsive noise, or speckle. These terms complicate the fringe detection algorithms and may result in measurement errors due to missing fringes.

Speckle is a specially relevant issue as far as OFI works typically using coherent sources focused onto diffusive targets. In order to keep track of speckle, Zabit et. al in [10] proposed the use of sliding window to determine the envelope of the OFI signal and locate the correct fringes for target reconstruction. Finding the envelope of the signal was performed twice - for positive and negative envelope separately. Another approaches have involved actively tracking of a bright speckle spot along time
[16]. Atashkhooei et. al [9] proposed two methods denoted "adaptive technique" and "sensor diversity technique" to deal with speckle effect and correct erroneous measurements. The main disadvantages of this technique is that the measurement requires the use of two OFI interferometers which need be synchronized. Often the case, the velocity of vibration of the target is usually measured in frequency domain by finding the Doppler shift in the signal. Kliese and Rakic [11] demonstrated mathematically and experimentally the broadening of Doppler spectrum due to speckle. Hence, even frequency domain analysis require some speckle compensation algorithmics in order to improve the accuracy of the measurements performed.

In this paper, we demonstrate the use of the continuous wavelet transform (CWT) to process OFI signals in time and frequency domains simultaneously for extracting target vibration parameters. The time domain is used for determining the target instantaneous displacement and time instance of direction reversal, while the frequency domain is used to determine the frequency and velocity of the vibration of the target. Unlike in Fourier analysis of OFI signals, here, the spectral characteristics of OFI signals are picked from the timefrequency plane evolved from CWT. The analysis of the time-frequency plane provided by the CWT is used to find the envelope of the OFI signal, and thus to keep track of speckle. The paper is organized as follows. In Sec.2 we will discuss the basics of the wavelet transform. Afterwards, in Sec.3 the methodology followed during the experimental process is described. Then Sec. 4 presents experimental results obtained with the proposed processing method. It is shown that the CWT can be used to remove OFI signal noise ina single stage, as well as to detect OFI fringes even in presence of speckle by taking advantage of the wavelet transform complex amplitude coefficients. Finally, we conclude the main contributions of this paper in Sec.5.

\section{Wavelet transform}

The Continuous Wavelet Transform (CWT) is a method of investigating time and frequency details of signals whose frequency content varies over time. They are specially suited, thus, to localize in time non-periodic perturbations or events. Wavelets are defined as localized waveforms which, instead of oscillating continuously, oscillate only for a given moment in time and rapidly decay to zero. The underlying property of wavelets is that they are localized both in time and frequency domain, unlike other types of bases such as the classical $\exp (j \omega t)$ used 
in the Fourier transform. Since OFI signals are dynamic in nature, wavelet transform is a very well suited tool for their analysis, allowing the retrieval of important parameters related to the vibration of a target.

The wavelet transform of a continuous signal $x(t)$ can be written as

$$
W(a, b)=\frac{1}{\sqrt{a}} \int_{-\infty}^{+\infty} x(t) \psi\left(\frac{t-b}{a}\right) d t,
$$

where $W(a, b)$ is the coefficient of the transform, $a$ represents the dilation (scale) wavelet parameter and $b$ represents the translation (shifting) wavelet parameter. The coefficient $W(a, b)$ is normalized by $1 / \sqrt{a}$ to ensure that all the wavelets have the same energy at every scale [12]. The scale parameter $a$ is inversely proportional to the frequency component of the analysed signal, and $b$ is related to the time instance at which a frequency component is generated. The physical interpretation of Eq.(4) is that $W(a, b)$ is obtained by correlating the scaled and shifted version of a mother wavelet $\psi(t-b) / a$ with $x(t)$. As $a$ is decreased, $\psi(t-b) / a$ is shrunk in time, thus, increasing the time resolution at the expense of decreasing the frequency resolution, and vice versa.

There exist a number of wavelet families and choosing one of them depends upon the application and nature of the information to be extracted. For the methods shown in this paper, we will use the Morlet complex wavelet transform, since it has shown good results in the analysis of sinusoidallike signals, a linear phase response, and a sampling dyadic nature [12]. Furthermore, we will show that the use of the complex wavelet coefficient allows the detection of fringes in an OFI signal as well as its envelope. The Morlet CWT is defined in time domain as

$$
\psi(t)=e^{i \omega_{0} t} e^{\frac{-t^{2}}{2}}
$$

The second term represents a rapidly decaying signal that constrains sine and cosine, represented in the first term, to a limited time. Fig. 2 shows the real and imaginary parts of Morlet wavelet that forms the basis of wavelet transform. Fig. 3 shows the effect of scaling on wavelet waveform in time and frequency domain. On reducing the scale by a factor of 8 (scale factor $1 / 8$ ) the wavelet in time domain shrinks and from time-frequency duality, the frequency is widen. Since the wavelet at low scale (dash) changes rapidly and has wide spectrum it is suitable to extract the information that varies rapidly with time and not with frequency. Inversely,

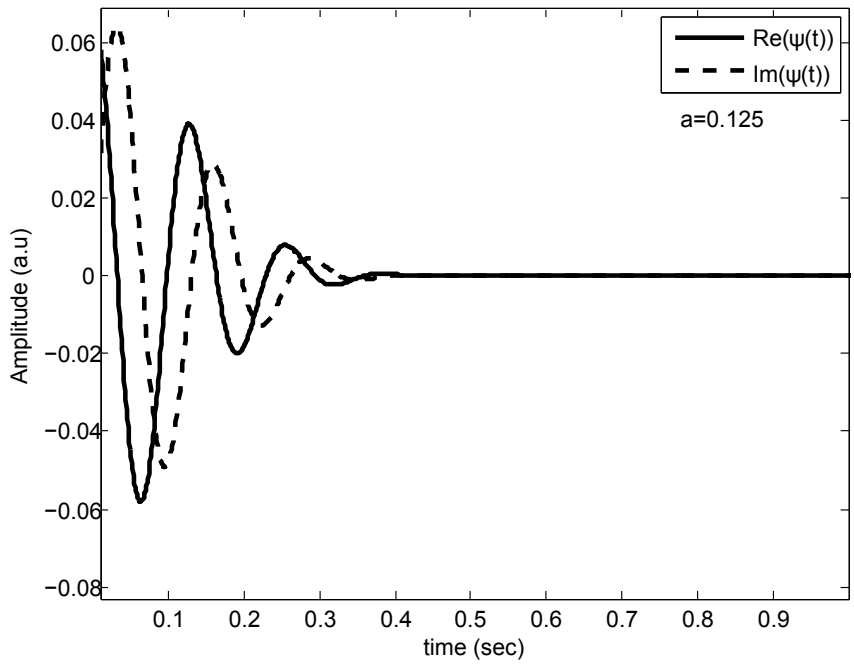

Figure 2. Real and Imaginary parts of Morlet Wavelet.
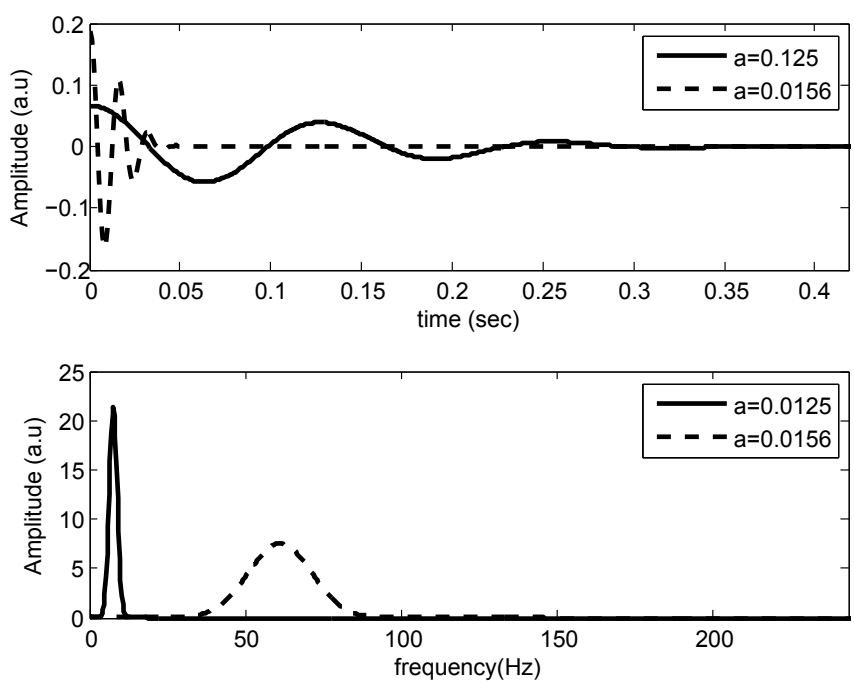

Figure 3. Effect of scale on Morlet wavelet time domain (up) frequency domain (down).

at larger scale (solid), the wavelet in time domain is extended and its corresponding spectrum is narrow. This property is used to extract the information that vary rapidly with frequency and not with time. Hence depending upon the situations of either requiring high temporal or spectral resolution, scale can be adjusted to retrieve the corresponding information. This makes wavelet transform a unique and advantageousness as compared to classical Fourier transform using sine and cosine as base function

In the frequency domain the complex wavelet 


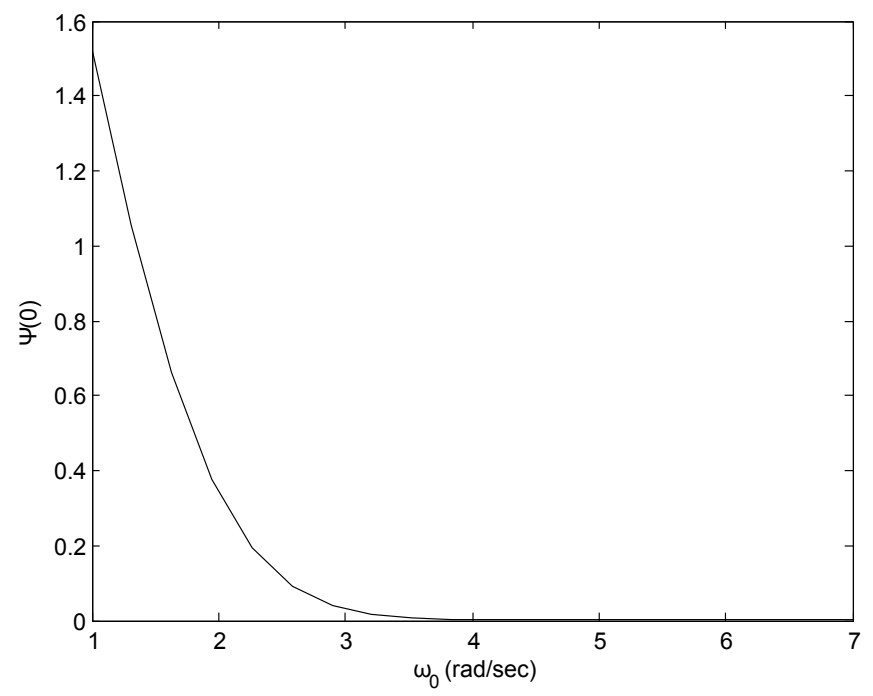

Figure 4. Admissibility condition for a wavelet.

transform is denoted as

$$
\begin{aligned}
\Psi(\omega) & =\int_{-\infty}^{\infty} \psi(t) e^{-j \omega t} d t \\
& =\sqrt{(2 \pi)} e^{\frac{-\left(\omega-\omega_{0}\right)^{2}}{2}}
\end{aligned}
$$

One of the admissibility conditions for a wavelet function requires it has no DC component. Hence, the Fourier transform of the complex wavelet should satisfy the condition $\Psi(0)=0$ [12]. From Eq.(7) and using above condition, we get

$$
\Psi(0)=\sqrt{2 \pi} e^{\frac{-\omega_{0}^{2}}{2}}
$$

The value of $\Psi(0)$ at different value of $\omega_{0}$ is shown in Fig. 4. Hence the value at which the admissibility condition i.e. $\Psi(0)=0$ is met at $\omega_{0} \geqslant 5$.

For this reason, along the remaining of the signal processing in this paper $\omega_{0}$ is chosen to be 6 , so it is the minimum value to accomplish the admissibility condition.

\section{Methodology}

\section{A. Experimental setup}

For the experimental results presented in the following section, a multiple quantum well AlGaAsP Hitachi HL6501 LD with a $0.65 \mu \mathrm{m}$ band was used. The emission wavelength $(\lambda)$ was measured using Instrument System's SPECTRO 320(D) R5 resulting in $692.5 \mathrm{~nm}$. The signal emitted by the LD was focused using a 352240-B Thorlabs aspheric lens with a fixed focal length and numerical aperture of 8 and $0.5 \mathrm{~mm}$ respectively. The lens was placed at a distance of $3.5 \mathrm{~mm}$ from the LD. A piezoelectric stage LISA P753.3CD with a maximum travel length of $38 \mu \mathrm{m}$ was used as target. It was placed at a distance of $12 \mathrm{~cm}$ from the $\mathrm{LD}$ in the configuration presented in Fig1. During the experiments, the target was vibrated by applying both a triangular and sinusoidal signal with amplitude $\left(A_{t}^{\prime}\right)$, and frequency $\left(f_{t}\right)$ of $2 \mathrm{~V}$ and $10 \mathrm{~Hz}$ respectively. The OFI signals were retrieved using a Tektronix DPO2024B oscilloscope. After the CWT signal processing, the measured displacement was compared with the measurements of the capacitive sensor embedded in the piezoelectric stage, which was used as reference. The experimental parameters are summarized in Table 1. Equivalent conditions and parameters were applied for obtaining a sinusoidal motion of the target.

\section{B. Signal processing}

The use of the wavelet transform for processing OFI signals presents a number of advantages over traditional, derivative based, peak detection methods. For instance, the use of wavelet transform allows signal noise removal and detection of OFI relevant parameters (such as the signal envelope, transitions, change of direction and frequency change) in the same processing step. Once this parameters are acquired, it is simple to estimate the related target vibration parameters.

A flowchart of the proposed processing method is depicted in Fig.5. The acquired signal is first subjected to a Morlet CWT. Due to the dyadic nature of Morlet CWT [13], it is computationally efficient to choose $a$ in terms of a power of 2 given by:

$$
a_{j}=a_{0} 2^{j d j}, \quad j=0,1,2 \ldots J-1,
$$

where $a_{0}$ is the smallest resolvable scale and it is set to the Nyquist frequency inverse and $d j$ is an increment in scale related to the expansion of the mother

Table 1. Experimental parameters

\begin{tabular}{lc}
\hline \multicolumn{1}{c}{ Parameters } & Value \\
\hline Distance of external target from laser $\left(L_{e x t}\right)$ & $12 \mathrm{~cm}$ \\
Voltage applied to Piezo (target) $\left(A_{t}^{\prime}\right)$ & $2 V(p p)$ \\
Target vibration amplitude (peak) $\left(A_{t}\right)$ & $7.6 \mu \mathrm{m}$ \\
Target vibration frequency $\left(f_{t}\right)$ & $10 \mathrm{~Hz}$ \\
Operating point of laser $\left(I_{o p}\right)$ & $45 \mathrm{~mA}$ \\
Emission wavelength of laser $\left(\lambda_{t h}\right)$ & $692.5 \mathrm{~nm}$ \\
Sampling time $(d t)$ & $8 \mu s$ \\
Sampling frequency $\left(f_{s}\right)$ & $125 \mathrm{kHz}$ \\
\hline
\end{tabular}




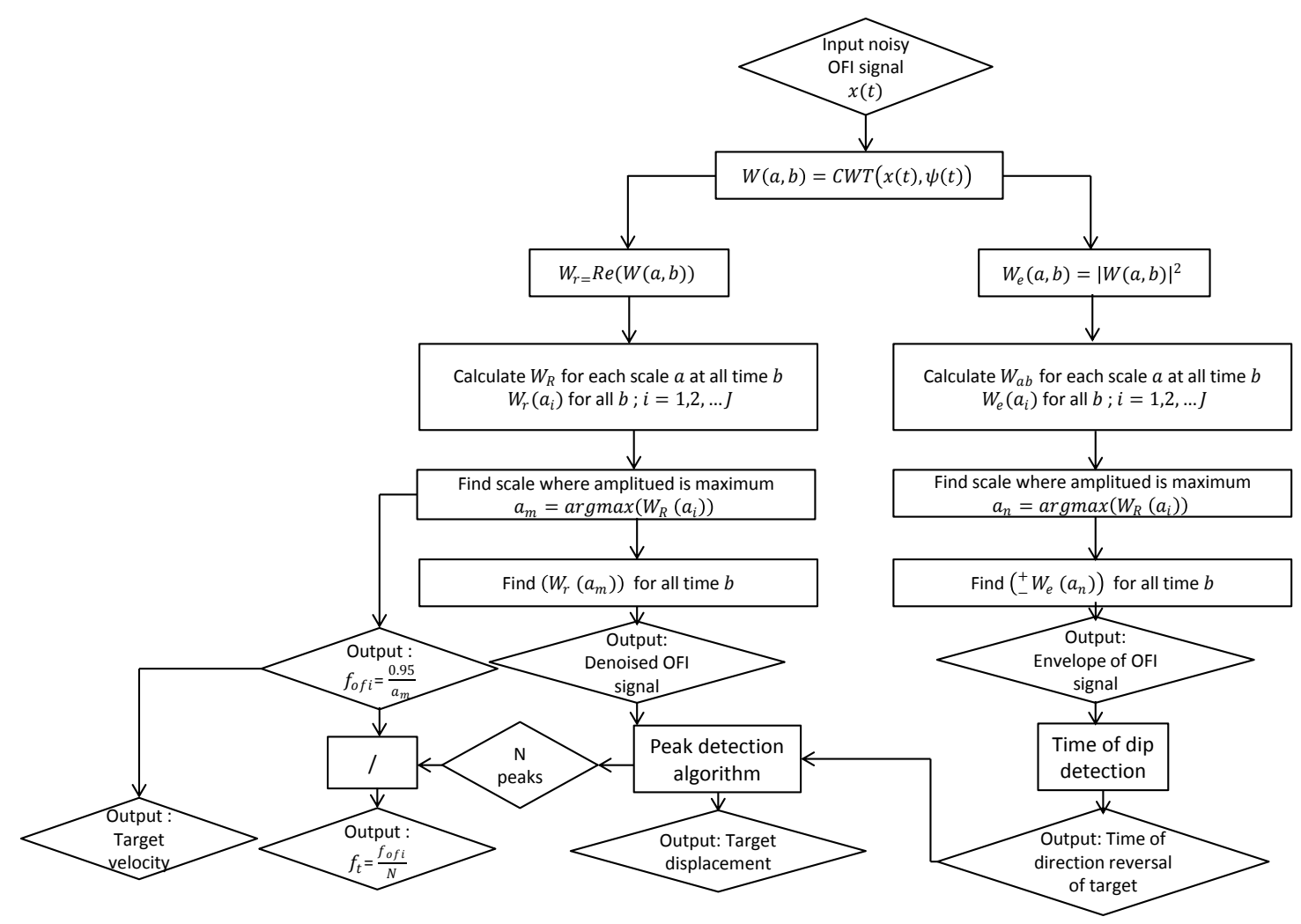

Figure 5. Flowchart for parameter extraction of vibrating target using wavelets.

wavelet. To balance the resolution and computing time, $d j$ was chosen to be $1 / 8$. The total scale $J$ can be found out by setting the frequency range $\left(f_{R}=f_{\max }-f_{\min }\right)$ to be analysed which determines the number of octaves $\left(N_{o}\right)$

$$
J=\frac{N_{o}}{d j}=\frac{1}{d j} \log _{2}\left(\frac{f_{\max }}{f_{\min }}\right),
$$

where $f_{\max }$ and $f_{\min }$ are the maximum and minimum analysed frequencies. An appropriate range of octaves and scales depends on the spectral content of the analysed signal, the highest requested CWT frequency and $d j$. In this paper, we selected the maximum CWT frequency to be equal to Nyquist frequency and frequency of analyzing mother wavelet to be $f_{0}=\omega_{0} /(2 \pi)$ where $\omega_{0}=6$ as discussed in Sec.2. The frequency $f_{a}$ corresponding to a scale $a$ is related to the central frequency $f_{0}$ of the wavelet by

$$
f_{a}=\frac{f_{0}}{a}=\frac{0.95}{a},
$$

A summary of the wavelet parameters used for OFI signal analysis in this paper is presented in Table
Table 2. Signal analysis parameter

\begin{tabular}{lc}
\hline \multicolumn{1}{c}{ Parameters } & Value \\
\hline Choice of wavelet & Morlet \\
Center frequency $\left(\omega_{0}\right)$ & $6 \mathrm{rad} / \mathrm{sec}$ \\
Minimum resolvable scale $\left(a_{0}\right)$ & $1.6 \times 10^{-5}$ \\
Where does it come from? Is it fixed & \\
Maximum frequency in CWT $\left(f_{\max }\right)$ & $62500 \mathrm{~Hz}$ \\
Scale increment $(d j)$ & $1 / 8$ \\
Total number of scale $(\mathrm{J})$ & 128 \\
\hline
\end{tabular}

2. After computing the Morlet CWT, the real part of $W(a, b)$ (further on noted $W_{r}(a, b)$ ) was plotted to visualize the spatial and temporal components of the OFI signal as shown in Fig.7. The Doppler shift caused by the target motion can be then easily estimated using the maximum value of $a$, noted $a_{\text {ofi }}$, shown on the scalogram (Fig.8). This Doppler shift will be obviously related to the target velocity as shown in Eq.(12).

Then, $W_{r}\left(a_{o f i}, b\right)$ is plotted for all time $b$ con- 
ditions, resulting in a de-noised OFI signal at the dominant scale. This waveform (Fig.9) is used as the basis for target displacement computation. Similarly, from the squared complex amplitude of $W\left(a_{o f i}, b\right)$ i.e $\left( \pm W_{e}\left(a_{o f i}, b\right)\right)$, the OFI signal envelope is obtained (Fig.9 dark solid). The signal envelope allows the determination of the time instance at which the target changes its direction. Each fringe on $W_{r}\left(a_{o f i}, b\right)$ (represented by a solid thin line in Fig.9) corresponds to a target displacement of $\lambda / 2$. The presence of a dip in the signal envelope is interpreted as a change of direction of the target, thus allowing to count all the fringes between two dips as displacements in the same direction. Hence, by counting the total number of fringes it is possible to estimate the target displacement and its trajectory.

Finally, the vibration frequency is calculated by dividing the obtained Doppler frequency by the total number of fringes in one period of the acquired OFI signal as shown in Eq.(12).

\section{Experimental results}

In this section the methodology proposed will be applied to an experimental OFI signal and we will show the results obtained by processing OFI signals with the proposed Morlet CWT method.

\section{A. Target velocity}

The experiment is performed using the parameters described in Table 1. Once the OFI signal (Fig.6) is acquired, it is subjected to a Morlet CWT. $W_{r}(a, b)$, and its corresponding scalogram, is then obtained. The scalogram shows the fraction of energy contained by a given frequency component at a par-
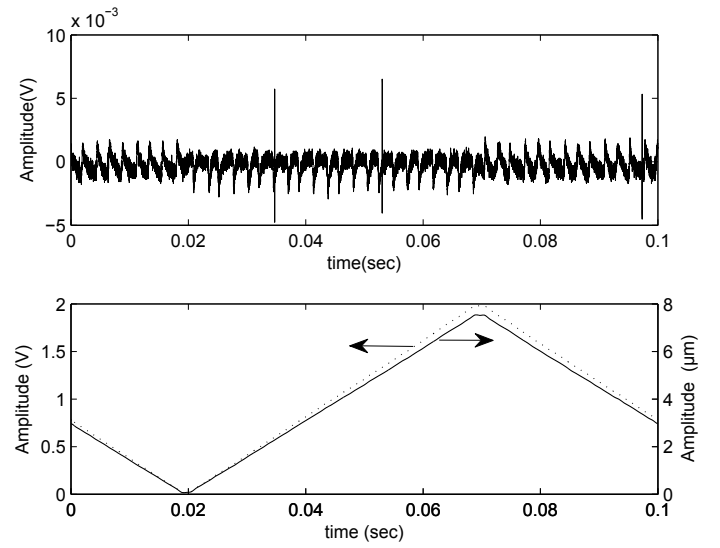

Figure 6. Top: OFI signal as a result of target vibration. Bottom: Voltage applied to the piezoelectric target (dotted line) and target motion (solid line).
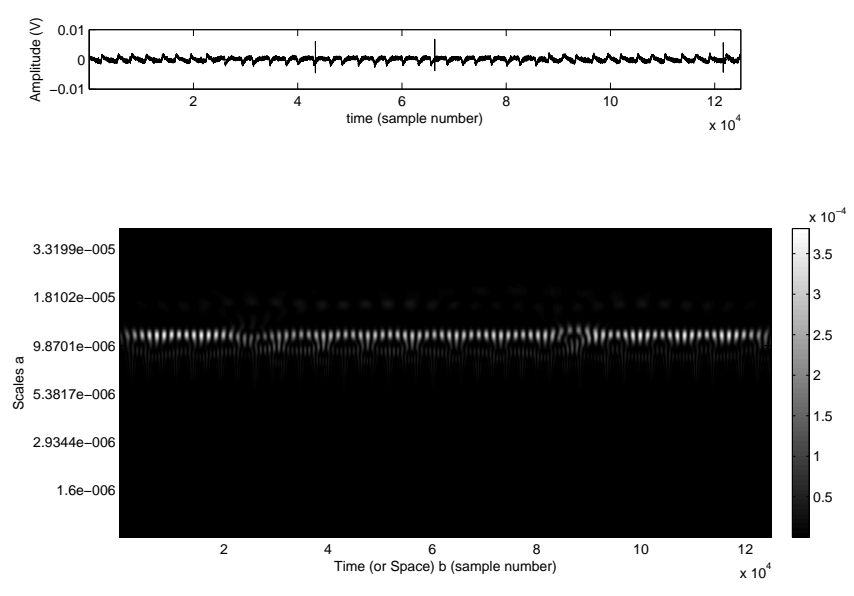

Figure 7. Top: OFI signal obtained for a triangular displacement. Bottom: Scalogram of the OFI signal.

ticular time instance of the OFI signal, as shown in Fig.7. It is also clear that the energy components of the noisy signal are localized at a particular scale, which is used to retrieve information about the dominant frequency of the OFI signal.

Fig. 8 shows $W_{r}(a, b)$ as a function of $a$. The maximum value of $W_{r}$ is found at $a=a_{\text {of } i}=0.002125$. When the value of $a$ is evaluated in Eq.(11), we obtain the dominant frequency in the OFI signal, which, for the proposed case, is $f_{\text {of } i}=447.1 \mathrm{~Hz}$. We can use this value in an straightforward manner to obtain the velocity of the vibrating target using the Doppler shift property. The OFI frequency is

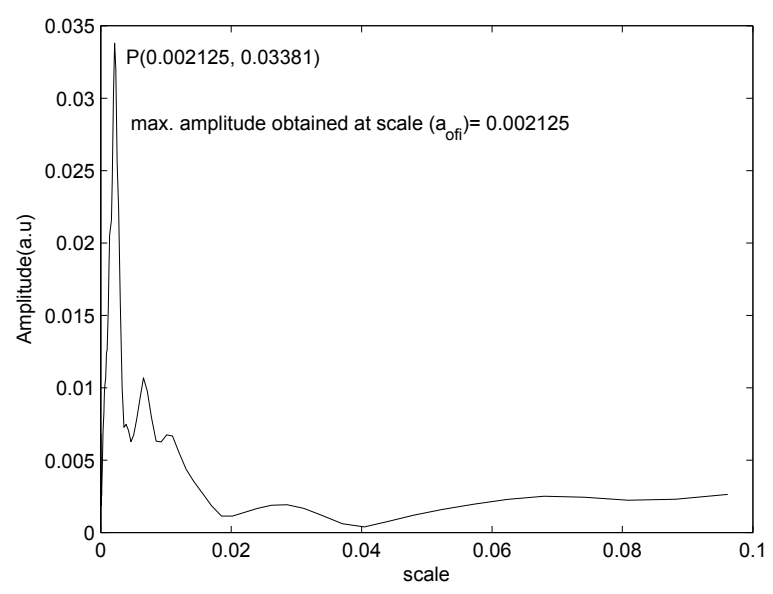

Figure 8. Real part of the complex wavelet coefficient plotted against the scale $\left(W_{r}\left(a_{o f i}, b\right)\right)$. At $a=a_{o f i}=$ $0.002125, W_{r}$ is maximum denoting a dominant frequency component $f_{\text {of } i}=447.1 \mathrm{~Hz}$. 
related to target velocity by:

$$
f_{D}=\frac{2 v}{\lambda}
$$

where $f_{D}$ is the Doppler shift, $v$ is the target velocity and $\lambda$ is the LD wavelength. If $f_{D}$ is substituted with $f_{\text {of } i}$ in Eq.(12), and using the $\lambda$ value defined in Table 1, the target velocity obtained equals $v=0.15 \mathrm{~mm} / \mathrm{s}$. This is in good agreement with the target velocity calculated from Fig.6, as the target moves linearly for $7.6 \mu \mathrm{m}$ during $0.05 \mathrm{~s}$, resulting in a velocity of $0.15 \mathrm{~mm} / \mathrm{s}$.

\section{B. Signal de-noising}

Experimental OFI signals typically contained white and impulsive noise, as is the case in our experimental signals. White noise, typically, is removed using low-pass filtering, and impulsive noise using a median filter [14][15]. The use of the wavelet transform enables to remove the effect of noise in the signal in a more efficient way, since it avoids the need of using two different filters and the associated signal processing, thus reducing the computational cost and time of the signal processing operation.

The wavelet transform is, in essence, a correlation between a scaled and shifted mother wavelet and a signal. Since noise itself is uncorrelated to the OFI signal, the resultant wavelet coefficients corresponding to noise are lower in magnitude than those corresponding to the signal. In order to retrieve the de-noised OFI signal, $W_{r}\left(a_{o f i}, b\right)$ is plotted resulting in Fig.9. As it can be seen, not only white and impulsive noise have been removed in a single step, but also signal processing and peak detection have been notably simplified.

\section{C. Envelope detection}

Speckle is an unwanted intensity modulation of the laser caused because of the finite size of the laser spot and irregularities greater than $\lambda / 2$ in the surface profile of the target. Speckle can cause the laser beam to add field amplitude constructively or destructively resulting in increase or decrease in the OFI signal amplitude and resulting in an error on the target displacement reconstruction, and is consubstantial to OFI due to its experimental conditions, with a coherent beam shone onto a rough target. Details on how speckle affect OFI signal can be found in [16].

To correctly detect, localize fringes and keep track of speckle induced fluctuations in signal amplitude, the envelope of the OFI signal is calculated using the square of the CWT magnitude $\left(W_{e}=\right.$

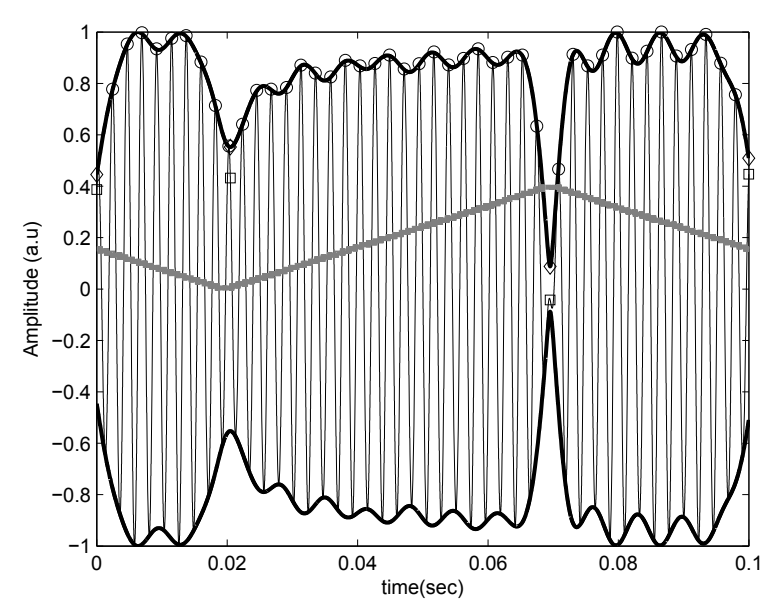

Figure 9. Envelope detection $W_{e}$ (dark black); Denoised OFI signal (black). Detected fringes (circle); Time instance of direction change (square); Scaled target motion (grey).

$\left.|W(a, b)|^{2}\right)$. This envelope detection of OFI signal not only helps to keep following the faded OFI signal due to speckle, but also enables to determine the time instance when the target motion changes its direction. Because of the wavelet correlation properties, when a target changes its displacement direction, the magnitude of the signal correlation decreases, resulting in lower wavelet coefficients which produce large fluctuations presented in dark solid in Fig.9.

The presented method is more computationally efficient than the sliding window metohd proposed by Zabit et. al in [10]. With $N$ elements and a $r$ window size is $O(N r)$ as compared to $O(N \log (N))$ for FFT based CWT. If the sample size is increased, the complexity of the FFT based CWT decreases as compared to the sliding window. In addition, to lower the complexity, it is only required to compute the CWT once to obtain both of the OFI signal envelopes resulting in computational gain of $2 r / \log N$ (factor 2 comes from fact that sliding window was performed twice), e.g. with a sample size of $N=125000$ and keeping window size of $k=5$, the computational cost for FFT based CWT is almost half as compared to the sliding window approach. Furthermore, as compared to the adaptive and sensor diversity techniques described [9], only one measurement is needed as compared to the two synchronous setups required to properly track speckle.

Furthermore, envelope detection can be used to determine the instant of target direction change. 
When the target motion (grey) Fig.9 reaches its maximum or minimum position, a rapid decrease of the envelope magnitude is detected. By calculating the time instance of this minimum (dip), it is possible to identify the time at which the target changed its direction. In Fig.9, the direction change is noted by the squares on the de-noised OFI signal. The circles, in the same figure, represent the time instance of detected OFI fringes.

\section{D. Target displacement}

After denoising the OFI signal and detecting the instance of target direction changes, it is possible to compute the target displacement. In this case, the peaks for each direction can be easily counted, thus, allowing the reconstruction of the target displacement profile. Since the OFI signal is almost symmetric, a simple fringe detection algorithm is implemented.

In the experiment, the OFI fringes were detected (Fig.9) and the target displacement was retrieved and compared against the reference capacitive sensor of the piezoelectric stage resulting in an absolute error value around $0.6 \lambda$. It should be noted we are only using fringe-counting method in a triangularshaped signal, so the moment in which the reversal of movement happens mayy induce significant errors.

To validate the accuracy of the approach, an equivalent analysis was performed using as a sinusoidal displacement of the target, with the parameters presented on Table 1 . In the case of the
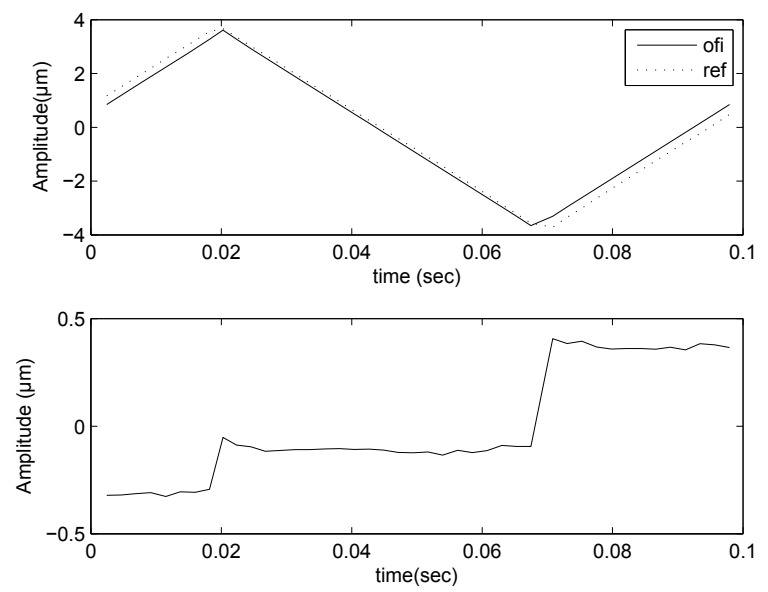

Figure 10. Target displacement retrieved using CWT processing on an OFI and comparison against capacitive reference sensor (top). Difference between OFI based and capacitive sensor (bottom) measurements.

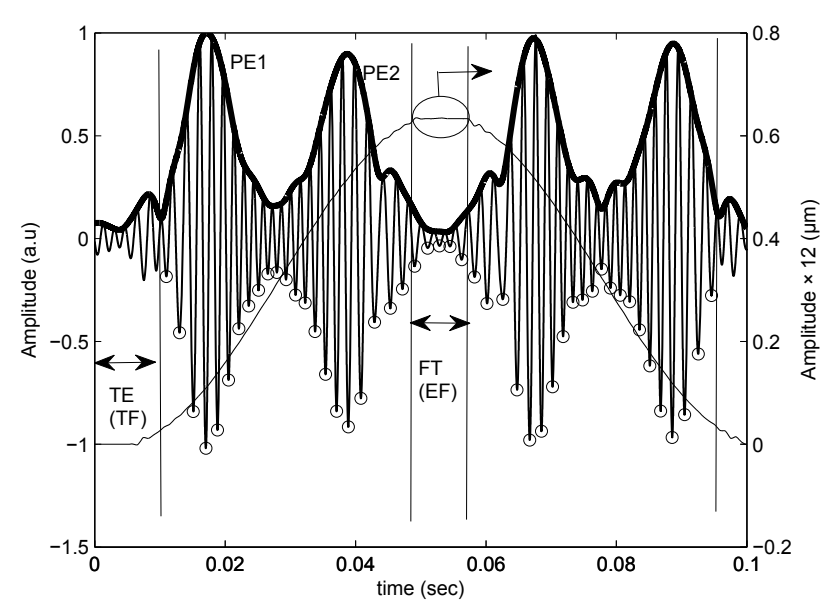

Figure 11. Different regions of the de-noised OFI signal and its envelope.

triangular motion, the change in direction occurs abruptly causing a narrow dip as shown in Fig.9. However, in the case of a sinusoidal target motion, because of the sine flat top, the transition time is not localized and there exists a finite time during which the transition occurs. Thus, for sinusoidallike motions, we talk about a time interval rather than a particular instance of time. These differences in nature between the sine and ramp like motions are picked-up by wavelet transform, and used to obtain an average error in displacement in the order of $0.1 \lambda$.

The de-noised OFI signal and its corresponding envelope for a sinusoidal target motion are shown in Fig.11. There are number of important conclusions that can be extracted from the signal envelope. First, the presence of a dip in the center corresponds to a discontinuity in the target motion. We denote region flat top $(F T)$, and fringes inside the FT region as error fringes $(E F)$. EF are very low in amplitude and is eliminated using a proper threshold. It is possible to detect two pulsed envelope zones (PE1,PE2) symmetric about FT where the OFI fringes are present. All the fringes in this region contribute to target displacement. Also, the envelope contains a tail at the edge denoted tail envelope $(T E)$ with its corresponding tail fringes $(T F)$, which, as in the case of EF, are not considered for displacement reconstruction as they represent the end or beginning of new cycle. A 'kink' at the tail denotes the fact that slope of target has significantly increased to cause effective fringes.

The displacement reconstruction and its comparison with the reference sensor of the piezoelectric 

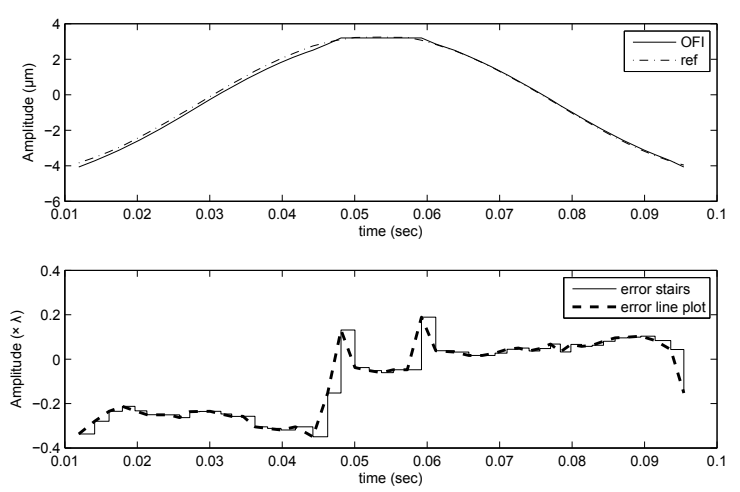

Figure 12. Top: Retrieved target displacement (solid line) compared with the reference capacitive sensor (dash-dotted line). Bottom: Resulting error swing within $0.2 \lambda$ and average error of $0.1 \lambda$ obtained from the difference of both sensor responses.

stage for the proposed experiment are shown in Fig.12. As it may be appreciated, the resulting error signal swing is within $0.2 \lambda$ with a mean error of $0.1 \lambda$.

\section{E. Target vibration frequency}

Once the target displacement is known, a Fourier transform of the displacement profile can be used to obtain the frequency of the target vibration $f_{t}$. However, this is an indirect method for calculating the aforementioned frequency. There are several situations (e.g. in condition monitoring of vibrating objects) where frequency is of more importance than the actual displacement. In such cases, it is possible to attain directly the frequency $f_{t}$ of a vibrating object dividing the OFI frequency by the number of fringes in one period of target motion $N$ as expressed in:

$$
f_{t}=\frac{f_{o f i}}{N} .
$$

Using the previously calculated values for the triangular motion $f_{o f i}=447.1 \mathrm{~Hz}$ and $N=43$, the target vibration frequency is estimated to be $f_{t}=10.4 \mathrm{~Hz}$ which is equal to original target vibration frequency as mentioned in Table 1. A similar analysis was performed using the Fourier transform method obtaining $f_{t}=10.0 \mathrm{~Hz}$. Thus, the wavelet based approach is consistent with Fourier analysis and with experimental data.

\section{Conclusion}

In this paper, we presented wavelet based signal processing to extract very efficiently different vi- bration parameters from a conventional OFI signal. The proposed methodology takes advantage of the wavelet properties to represent OFI signal in time and frequency domain simultaneously, allowing noise filtering and signal processing in a single processing step . The use of the Morlet CWT enabled to remove white and impulsive noise present in the OFI signal, and to extract target vibration parameters (e.g. amplitude, frequency, velocity). Furthermore, the use of Morlet CWT to recover the envelope of OFI signals proved to be an advantage in those cases where speckle induced signal fading. The method is computationally very efficient as compared to envelope detection to track speckle, or to the use of adaptive and sensor diversity techniques. It was also demonstrated that wavelet analysis permits to precisely pick and detect the occurrence of OFI fringes, hence, allowing a better accuracy in target displacement retrieval. The average error found out for sinusoidal type motion is in the order of $0.1 \lambda$. As a whole, we have demonstrated that wavelet analysis is a well suited, simple and efficient technique for processing and studying the spectral and spatial features of OFI signal dynamics, specially when transients are involved.

\section{Acknowledgement}

The authors would like to thank the Spanish Ministry of Science and Innovation for the funding provided by Plan Nacional $\mathrm{I}+\mathrm{D}+\mathrm{I}$ through project DPI2011-25525. Ajit Jha would like to thank European Union funding via Erasmus Mundus programme for the fellowship. Francisco J. Azcona would also like to thank AGAUR for its funding through pre-doctoral grant 2012FI_BI 00240. Carlos Yanez would like to thank UPC-Barcelonatech for its funding through his FPU pre-doctoral grant.

\section{References}

[1] R. Lang and K. Kobayashi, "External optical feedback effects on semiconductor injection laser properties," IEEE J. Quantum Electron. 16, 347-355 (1980).

[2] Y. Yu, G. Giuliani, and S. Donati, "Measurement of the linewidth enhancement factor of semiconductor lasers based on the optical feed-back self-mixing effect," IEEE Photon. Technol. Lett. 16, 990-992 (2004).

[3] K. Petermann, "External optical feedback phenomena in semiconductor lasers," IEEE J. Sel. Topics Quantum Electron. 1, 480-489 (1995).

[4] G. Plantier, C. Bes, and T. Bosch, "Behavioral model of a self-mixing laser diode sensor," IEEE J. Quantum Electron. 41, 1157-1167 (2005). 
[5] G. Acket, D. Lenstra, A. D. Boef, and B. Verbeek, "The influence of feedback intensity on longitudinal mode properties and optical noise in index-guided semiconductor lasers," IEEE J. Quantum Electron. 20, 1163-1169 (1984).

[6] D. M. Kane and K. A. Shore, Unlocking Dynamical Diversity Optical Feedback Effects on Semiconductor Lasers (John Wiley and Sons Ltd., 2005).

[7] Y. Yu, J. Xi, and J. F. Chicharo, "Measuring the feedback parameter of a semiconductor laser with external optical feedback," Opt. Express 19, 95829593 (2011).

[8] S.Donati, G.Giuliani, and S.Merlo, "Laser diode feedback interferometer for measurement of displacements without ambiguity," IEEE J. Quantum Electron. 31, 113-119 (1995).

[9] R. Atashkhooei, S. Royo, and F. Azcona, "Dealing with speckle effects in self-mixing interferometry measurements," IEEE Sensors J. 13, 1641-1647 (2013).

[10] U. Zabit, O. D. Bernal, and T. Bosch, "Self-mixing laser sensor for large displacements: Signal recovery in the presence of speckle," IEEE Sensors J. 13, 824-831 (2013).

[11] R. Kliese and A. D. Rakic, "Spectral broadening caused by dynamic speckle in self-mixing velocimetry sensors," Opt. Express 20, 18757-18771 (2012).

[12] M.Farge, "Wavelet transforms and their applications to turbulence," Annu. Rev. Fluid Mech. 24, 395-457 (1992).

[13] P. Goupillaud, A. Grossman, and J. Morlet, "Cycleoctave and related transforms in seismic signal analysis," Soc. of Expl. Geophys. 23, 85-102 (1984).

[14] Y. I. Kazuyoshi Itoh and T. Minami, "Nearestneighbor median filter," Appl. Opt. 27, 3445-3450 (1988).

[15] P. S. Windyga, "Fast impulsive noise removal," IEEE Trans. Image Process. 10, 173-179 (2001).

[16] S. Donati, G. Martini, and T. Tambosso, "Speckle pattern errors in self-mixing interferometry," IEEE J. Quantum Electron. 49, 798-806 (2013). 